\title{
How to Set Up a Perioperative BNP/NTproBNP and Troponin Screening Program
}

Erin N. Sloan, MD, Erin E. Morley, MD, FRCPC

The Division of Internal Medicine, University of British Columbia, Vancouver, BC, Canada

Corresponding author: ensloan@gmail.com

Submitted: November 9, 2020. Accepted: January 18, 2021. Published: March 16, 2020. DOI: 1022374/cjgim.v16iSP1.531.

\section{Key Learning Points}

1. Review the rationale and evidence to support the use of perioperative biomarkers (BNP/ NTproBNP and troponin)

2. Explore three unique models for integrating perioperative biomarkers into practice

3. Apply guiding principles to perform a site-specific assessment of the current approach to perioperative risk assessment and review strategies on how to initiate implementation of a perioperative biomarker screening program

4. Understand commonly faced challenges, and proposed solutions, for implementing a perioperative biomarker screening program

5. Appreciate the potential role for a shared-care model to facilitate perioperative biomarkers screening and management

\section{Points à retenir}

1. Examiner la justification et les données probantes à l'appui de l'utilisation de biomarqueurs périopératoires (BNP/NTproBNP et troponine).

2. Explorer trois modèles uniques pour l'intégration de biomarqueurs périopératoires dans la pratique.

3. Appliquer des principes directeurs pour réaliser une évaluation propre au site de l'approche actuelle en matière dévaluation des risques périopératoires et examiner les stratégies sur la manière d’entreprendre la mise en ouvre d'un programme de dépistage périopératoire de biomarqueurs.

4. Comprendre les défis couramment affrontés et les solutions proposées pour la mise en œuvre d'un programme de dépistage périopératoire de biomarqueurs.

5. Prendre la mesure du rôle potentiel d'un modèle de partage des soins pour faciliter le dépistage et la prise en charge de biomarqueurs périopératoires.

Over 300 million adults worldwide undergo noncardiac surgery annually, ${ }^{1}$ and over 10 million patients will suffer a major cardiac complication. ${ }^{2}$ The magnitude of this problem is likely to increase due to an aging population, an increase in the prevalence of cardiovascular disease, and a trend toward more surgical interventions in elderly patients.

The 2014 American College of Cardiology (ACC) and American Heart Association (AHA) noncardiac surgery guidelines encourage the use of postoperative ECG and troponin in the setting of signs or symptoms of ischemia, stating that the usefulness of routine postoperative screening is uncertain in the absence of established risk and benefits of a defined management strategy. ${ }^{3}$ There is no mention of preoperative risk assessment using brain natriuretic peptide (BNP) or N-terminal pro-BNP-type natriuretic peptide (NTproBNP). In contrast, the European Society of Cardiology and European Society of Anesthesiology, also published in 2014, notes that preoperative BNP/NTproBNP levels have prognostic value for cardiac events after major noncardiac vascular surgery and suggest testing of cardiac troponin in high-risk patients, both before and 48-72 $\mathrm{h}$ after major surgery. ${ }^{4}$ More recently, the 2017 Canadian Cardiovascular Society (CCS) perioperative guidelines recommend the use of perioperative biomarkers including preoperative BNP/NTproBNP testing to identify patients at a 
higher risk for perioperative adverse cardiac events, and routine troponin monitoring postoperatively to detect myocardial ischemia in these higher risk patients. ${ }^{5}$ While perioperative biomarker screening has not been compared to usual care in a randomized controlled trial (RCT), there is increasing evidence to support its role in both risk assessment and monitoring of higher risk patients undergoing noncardiac surgery.

Elevated preoperative levels of BNP/NTproBNP are strongly associated with vascular death and myocardial injury after noncardiac surgery (MINS) within 30 days of noncardiac surgery ${ }^{6,7}$ Although the Revised Cardiac Risk Index (RCRI) is a widely validated model for cardiac risk stratification, ${ }^{5,8,9}$ the addition of preoperative BNP/NTproBNP testing serves to further refine perioperative cardiovascular risk prediction. ${ }^{6,710}$

Most MINS events occur within $48 \mathrm{~h}$ of surgery, and the majority of these events are clinically silent. The VISION study showed that without routine troponin monitoring, $84 \%$ of the MINS events would be undetected. ${ }^{2}$ Detecting MINS is clinically important as, compared to patients without MINS, patients have an almost ninefold increased risk for major adverse cardiac events (MACE) and death at 30 days ( $9.8 \%$ vs $1.1 \%$ ). ${ }^{2,11}$ This increased mortality risk was confirmed in two cohort studies. ${ }^{12,13}$

Diagnosis of MINS allows for optimization of medications and cardiac testing to improve cardiac outcomes. ${ }^{14-16}$ An economic analysis supporting postoperative troponin monitoring is costeffective. ${ }^{17}$ By comparison, the screening costs for breast or cervical cancer detection is $>10$-fold the cost to detect MINS. Given that patients with MINS are at risk of dying within 30 days, this highlights the importance of screening.

Given considerable variability in Canadian health care centers with regard to size, staffing, local expertise, rural versus urban setting, surgical diversity, and volume, it is unlikely that a single approach for perioperative biomarker screening can be successfully implemented at every hospital. Three models successfully being used in British Columbia and Ontario are highlighted below, along with suggestions on how to overcome common implementation challenges and a general appraisal tool that can be tailored to suit one's unique clinical environment.

\section{The Vancouver General Hospital Model}

The Vancouver General Hospital, an academic, tertiary care referral center for British Columbia and the Yukon Territory, is the second largest hospital in Canada with 17-19 operating rooms in use per day. This hospital performs on average 20,000 surgical procedures annually, of which approximately $70 \%$ are inpatient procedures and approximately 1000 are cardiac surgeries. Nearly $30 \%$ of the surgical cases are unscheduled. VGH uses BNP and troponin I (TnI) but will change to NTproBNP in January 2021 with plans to transition to high-sensitivity troponin T (HSTnT).
In 2015, the Internal Medicine Perioperative Consult Team (IMPCT) was created. The IMPCT service is staffed by 11 general internal medicine (GIM) staff physicians with an interest or expertise in perioperative medicine. In discussion with hospital administration and surgical and anesthesiology clinicians, the IMPCT team started with providing shared postoperative care for all hip fracture patients over age 65 , and on a consultation basis for patients undergoing hepatobiliary, vascular, or major urological surgery. Initially, to ensure that the IMPCT service would be financially viable, high-risk patient criteria were established to identify who would receive consultations. IMPCT also provided preoperative consultations in the post-anesthesia care unit (PACU) on two half-days per week.

The service was well received by surgical and anesthesia colleagues and subsequently expanded to two separate lines of IMPCT, each comprising a staff internist with one or more GIM fellows, a GIM resident, and a surgical resident. Preprinted order sets (PPO) were designed to identify patients appropriate for preoperative BNP testing (Figure 1) and postoperative troponin monitoring. The order sets included instructions for "what to do" and "who to call" if the troponin was elevated (Figure 2). The PPOs along with a summary of the Canadian Cardiovascular Society guidelines were presented to the surgical executive committee. Once approved, each surgical service was offered a dedicated rounds presentation on perioperative cardiac risk, which also served to field questions and to elicit feedback specific to each surgical population. The nurses in the post-anesthesia recovery unit (PACU) and on the surgical wards are another important stakeholder group and should be included as they often implement the PPOs.

At present, BNP may be ordered by the surgeon (some have incorporated into their electronic medical record directly with routine preoperative blood work), by anesthesia in the PAC (all BNP results are followed up by the PAC physician lead), or by the IMPCT team member if the patient is seen preoperatively. An elevated preoperative $\mathrm{BNP}(\geq 92 \mathrm{ng} / \mathrm{L})$ is evaluated in the context of each patient's medical history and physical examination. Most patients with a mild elevation (i.e., BNP of $150 \mathrm{ng} / \mathrm{L}$, asymptomatic, and satisfactory physical examination in the context of the patient's known medical issues) do not require further investigations prior to surgery. However, as these patients are still at a higher risk of perioperative MACE, their names are added to the electronic IMPCT inpatient list to be seen on postoperative day one (or immediately postoperatively if there are concerns from surgery or anesthesia). Patients who have an unexpected degree of elevation in preoperative BNP/NTproBNP (i.e., BNP $400 \mathrm{ng} / \mathrm{L}$, with no known comorbidities such as established cardiovascular or renal disease) are scheduled to have a preoperative IMPCT consultation and may warrant further investigations besides a clear discussion of perioperative cardiac risk. 


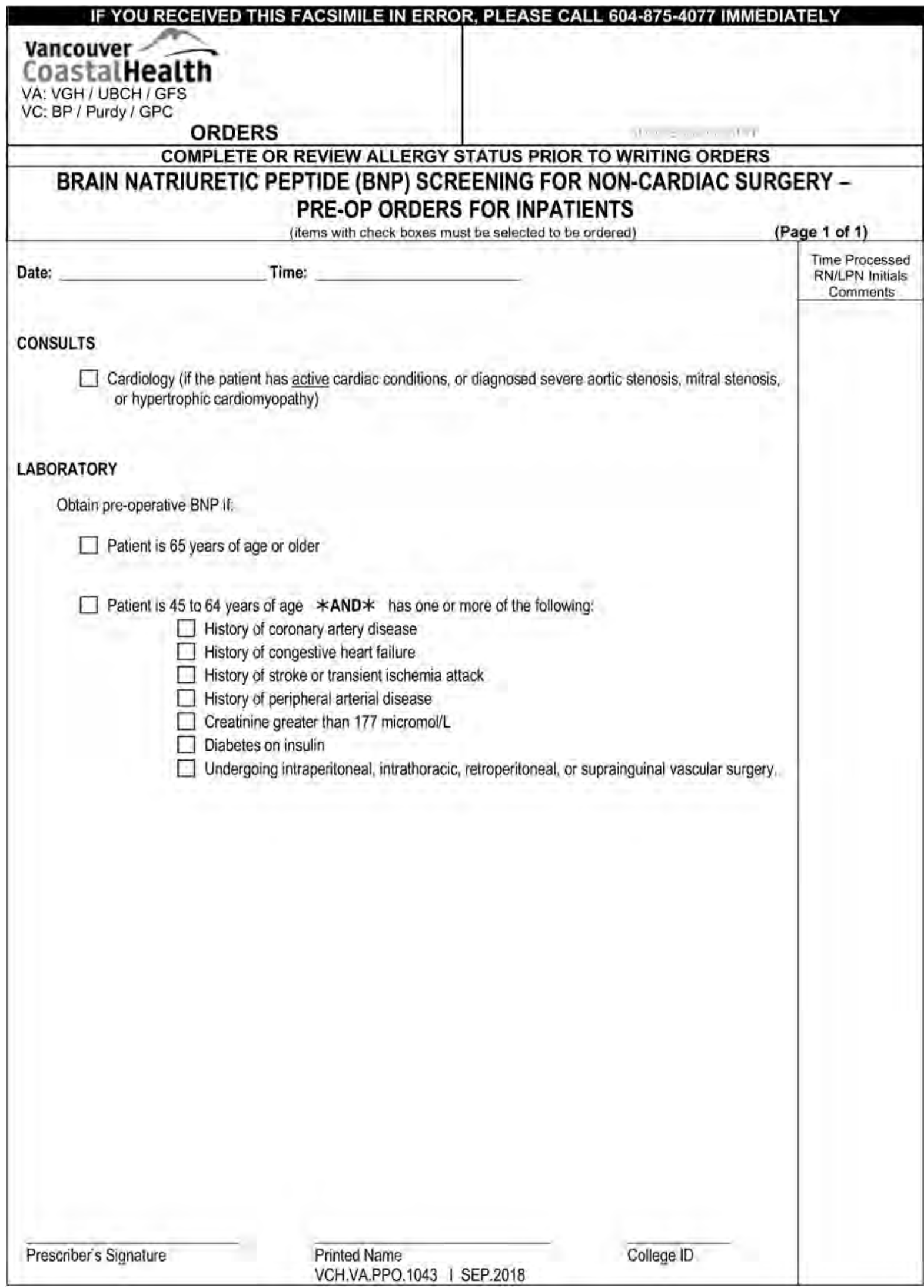

Figure 1. Brain Natriuretic Peptide (BNP) Screening for Non-cardiac Surgery - Pre-op Orders for Inpatients. 
IF YOU RECEIVED THIS FACSIMILE IN ERROR, PLEASE CALL 604-875-4077 IMMEDIATELY

\section{Vancouver \\ CoastalHealth}

VA: VGH / UBCH / GFS

VC: BP / Purdy / GPC

ORDERS

COMPLETE OR REVIEW ALLERGY STATUS PRIOR TO WRITING ORDERS

MYOCARDIAL INJURY AFTER NON-CARDIAC SURGERY (MINS) - POST-OP SURVEILLANCE ORDERS

(items with check boxes must be selected to be ordered)

Date: Time:

Pre-operative BNP

or NT-proBNP

If Pre-operative BNP less than $92 \mathrm{ng} / \mathrm{L}$ or NT-proBNP less than $200 \mathrm{ng} / \mathrm{L}$ :

no empiric ECG or troponin monitoring required unless otherwise clinically indicated

If Pre-operative BNP $92 \mathrm{ng} / \mathrm{L}$ or greater, or NT-proBNP $200 \mathrm{ng} / \mathrm{L}$ or greater $* \mathrm{OR} *$ if no preoperative BNP or NT-proBNP available but patient has one or more of the following:

Age 65 years or older

Coronary artery disease

Heart failure

Creatinine more than 177 micromol/L

Diabetes on Insulin

Stroke or TIA

Then obtain:

Undergoing intraperitoneal, intrathoracic, retroperitoneal, or suprainguinal vascular surgery

(Page 1 of 1)

Time Processed

RN/LPN Initials

Comments

$\square$ ECG on arrival in Post Anesthesia Care Unit (PACU)

Troponin on arrival in PACU \& on postoperative days 1,2 and 3

Notes to Prescriber

If troponin is positive

Order and review ECG, assess patient

Consult Internal Medicine Perioperative Consult Team (IMPCT) if:

Troponin greater than 0.03 but less than 1 , and no new ECG changes suggestive

of ischemia (e.g. T-wave inversion, ST depression, ST elevation)

No chest pain

Consult Cardiology if:

Troponin is equal or greater than 1

New ECG changes suggestive of ischemia (e.g. T-wave inversion, ST depression, ST elevation)

Chest pain

Figure 2. Myocardial Injury after Non-cardiac Surgery (MINS) -Post-op Surveillance Orders. 


\section{Myocardial Injury after Non-Cardiac Surgery (MINS) Surveillance Flow Diagram}

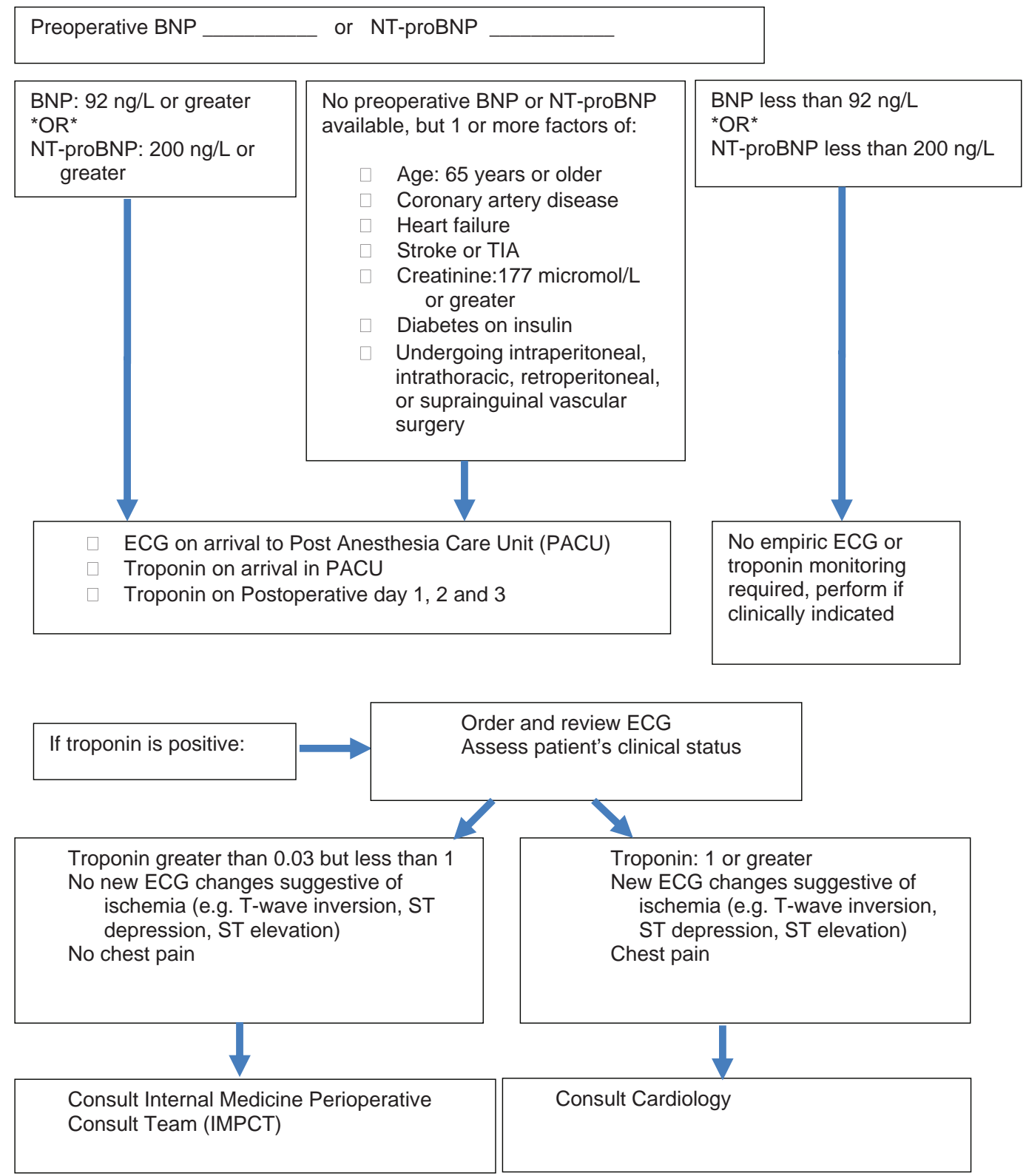

Figure 2. (Continued)

Postoperatively patients are discharged from the PACU to a regular surgical ward bed, surgical step-down bed, or the ICU as appropriate. The surgeon remains the most responsible physician (MRP). IMPCT provides medical co-management for patients with complex medical comorbidities and assessment for patients with elevated postoperative troponin values (ordered based on elevated preoperative BNP). Via close communication with the surgical team, for patients with elevated troponin postoperatively, pharmacological intervention for MINS (i.e., ASA, statin) and/or other strategies to reduce ischemic burden (i.e., optimization of hemoglobin, fluid status, hold offending medications) are considered. Cardiology consultations are reserved for patients who have evidence of active ischemia (i.e., ischemic symptoms, ischemic ECG changes, or arbitrarily a TnI of 1.0 or higher, as determined in consensus with cardiology and as detailed on the PPOs. IMPCT follows inpatients while acute medical issues exist and can facilitate outpatient follow up as needed. Overnight calls are managed primarily by the surgical team with IMPCT support in urgent situations. In house critical care outreach, or rarely, the internal medicine clinical associate can provide support overnight as required. 
The COVID-19 pandemic has modified the delivery of outpatient preoperative assessment. The IMPCT team's patient volume is unchanged, but assessment is provided via phone while anesthesia uses zoom for virtual consultations. A nurse subsequently contacts patients for COVID-19 screening and to arrange for blood work (including BNP), and an outpatient ECG is to be done at the patient's local lab.

Surgical patient co-management provided by IMPCT has been an essential part of implementing perioperative biomarker testing, and this model may confer addition benefits for patients, colleagues, and budgetary considerations. While co-management has not shown universal improvement in outcomes, ${ }^{18}$ metaanalyses of shared care by geriatricians and orthopedic surgeons have showed decreased mortality and shorter length of stay (LOS). ${ }^{19,20}$ Similarly, a meta-analysis of surgical patients with IM co-management demonstrated decreased LOS and mortality. ${ }^{18}$ An RCT looking at co-management by hospitalists and surgeons versus a consultation care model showed decreased minor, but not major, complications and fewer discharge delays. ${ }^{21}$ Studies have also shown that shared care models increase prescribing of evidence-based treatment, ${ }^{22}$ increase surgeon and nurse satisfaction, ${ }^{21}$ decrease $\operatorname{LOS}^{19,20,23}$, and decrease readmissions resulting in overall cost savings. ${ }^{23}$

\section{The St. Paul's Hospital Model, Vancouver}

St. Paul's Hospital (SPH) is an academic, tertiary care center and referral center for British Columbia and the Yukon Territory. With 16 operating rooms are in use per day, this hospital performs over 12,500 surgical cases per year, of which approximately half are inpatient surgeries and approximately 1250 are cardiac surgeries. Over $17 \%$ of the surgical cases are unscheduled.

The anesthesiologists at SPH initiated guideline-driven care, in collaboration with internal medicine, by implementing perioperative biomarker use in accordance with the CCS guidelines. While an internal medicine inpatient consult service exists, it is not a dedicated perioperative service. Surgeons or anesthetists may refer patients to the internal medicine rapid access clinic for a preoperative consultation, or in the absence of complex medical issues, patients may have preoperative NTproBNP testing ordered by the anesthetist in the PAC. Due to COVID-19, anesthesiology provides virtual assessments for most patients.

On the day of the surgery, the perioperative anesthesiologist ensures that patients have had NTproBNP testing as appropriate. If indicated, but not yet performed, NTproBNP is either added to other recent blood work or drawn preoperatively. In patients with an NTproBNP > $200 \mathrm{ng} / \mathrm{L}$, postoperative troponin levels and ECG testing are ordered electronically. In the absence of surgical stepdown units at St. Paul's, patients with NTproBNP $>1500 \mathrm{ng} / \mathrm{L}$ are monitored in the anesthesia-run surgical high acuity unit for at least $24 \mathrm{~h}$ postoperatively. On the regular surgical wards, patients with elevated preoperative NTproBNP remain on the anesthesia perioperative list to facilitate monitoring of postoperative troponin levels. The surgeon continues as the MRP and overnight calls are managed by the surgical service. Patients with elevated troponin levels are assessed on the surgical ward by the perioperative anesthesiologist, and internal medicine is consulted for evaluation, risk stratification, and follow-up care. To assist in patient management, a GIM vascular medicine clinic provides three half-day clinics per week to manage postoperative complications such as MINS with in-person or virtual patient visits.

\section{The Juravinski Hospital Model, Hamilton}

Located in Hamilton, Ontario, and affiliated with the Population Health Research Institute, the Juravinski Hospital was one of the first centers to implement perioperative biomarker measurements as part of routine perioperative management. With nine operating rooms were used daily, this hospital performs over 6500 noncardiac surgeries annually, of which nearly $70 \%$ are inpatient procedures and $26 \%$ are considered urgent. Initially developed as a cardiology-run service with focus on preoperative evaluation and postoperative cardiac events, this model of care evolved from consultants working in isolation, to a dedicated perioperative service comprising GIM and cardiology services, working closely with the anesthesiology service. NTproBNP is typically ordered by the anesthesiology service during the preoperative assessment and patients with NTproBNP $>100 \mathrm{ng} / \mathrm{L}$ are referred to this perioperative service. Pre-printed order sets are used to ensure that postoperative monitoring is ordered appropriately

Until recently, preoperative NTproBNP testing was not available as an onsite test with timely results. The availability of point-of-care (POC) NTproBNP testing allowed for rapid, individualized patient assessment. Limitations of POC NTproBNP testing include the need for a specific handheld device for each provider or shared clinic, the cost per test being $\$ 15.00-22.00,{ }^{24}$ and integrating the results into the patient's electronic medical record (though some systems report seamless integration with hospital computer systems). ${ }^{25}$ Prior to integrating POC results electronically, establishing accuracy with a laboratory based NTproBNP and related quality control measures would be required. At the very least, the NTproBNP result can be highlighted in a dictated consultation note. Due to COVID-19, preoperative assessments are often performed remotely and preoperative NTproBNP may be less readily available. POC NTproBNP immediately prior to the OR may be an option to help guide the need for postoperative troponin monitoring.

The Juravinski perioperative inpatient service is staffed by a GIM or cardiology attending, a nurse practitioner (NP), and a resident/ fellow. The service provides GIM consultative support including 
atrial fibrillation, heart failure, and MINS. Other cardiology concerns are referred to the cardiology consult service. The surgeon remains the MRP, and overnight concerns are directed to the surgical team with infrequent involvement of the IM senior resident. Prior to COVID-19, the preoperative clinic ran 5 days/week (now 3 half days) and postoperative clinic started at 1 half day/week and now runs 3 half days/week with demand to expand further.

\section{A Tailored Approach to Preoperative BNP/ NTproBNP and Postoperative Troponin Screening}

As discussed, implementing a perioperative biomarker screening program follows the 2017 CCS guidelines. Determining the right approach requires a multifaceted evaluation of current workplace culture; key stakeholders; local interest and expertise; and identification of short-, medium-, and long-term goals. A local champion(s) who can drive the process is essential. To facilitate the analysis of a center's current approach and for strategies on how to implement a perioperative biomarker screening program, consider the following questions:

\section{Assess Current Culture}

- Aside from the surgeon, who else is providing perioperative care?

- What risk stratification tools are currently being used? What are the validity, availability, and cost of these tools?

- Who could order preoperative BNP/NTproBNP (i.e., surgeon, anesthesia, or IM)?

- How could BNP/NTproBNP be ordered (i.e., add to surgeon's routine preoperative blood work on EMR, creation of PPO, anesthetist to request for blood work in PAC, or POC testing in surgeon's office, PAC, or immediately prior to surgery)?

- Who would follow up on the BNP/NTproBNP result? Is there an established referral pathway for abnormal values (i.e., are timely perioperative medicine consults available)?

- How are patients at an increased risk of postoperative MACE being identified and monitored?

- What capacity exists to see patients with postoperative troponin elevation in a timely manner? If limited, what would need to change to facilitate this important step? Is there a role for a nonphysician (i.e., NP or Physician Assistant (PA)) to help with staffing and workload?

- What follow-up options exist locally or virtually for patients with postoperative complications such as MINS?

- What aspects of preoperative and postoperative care are working well?

- Where is the room for improvement?

\section{Identify Key Stakeholders, Including but Not Limited To}

- Anesthesia

- Surgery
- Internal medicine

- Cardiology

- Geriatrics

- Primary care provider

- Laboratory medicine

- Nurses (surgical screening, PACU, surgical ward)

- Hospital administration

- Patient care office

- Quality improvement office

- Physician engagement groups (is funding available to help facilitate an interdisciplinary team?)

\section{Assess Level of Local Expertise and Interest}

- Are the key stakeholders familiar with the CCS perioperative guidelines?

- If not, what would be required to get all of the key stakeholders familiar with and motivated to implement guideline-driven care (i.e., grand rounds, guest speaker, CME event)?

- Who could serve as a "champion" from key disciplines to steer a committee?

- Are there any obvious barriers (i.e., time constraints, interpersonal issues, territorial services, knowledge deficits, funding issues)?

- Securing physician engagement funding enhances meeting attendance, helps physicians feel their time and their expertise is valued, promotes team building, and enhances collegiality across disciplines. $^{26}$

\section{Determine a Starting Point and Consider Medium to} Long-Term Goals

- What is needed right now to improve patient safety and care? If implementing a system hospital wide is daunting, consider a select group of higher risk surgical patients as a starting point.

- Who is available to assist with this project right now? How much bandwidth do they have? Are scheduling or funding changes required to increase the likelihood of success? Is dedicated training required?

- Start small and expand in time.

- Medium- to long-term goals: consider recruiting physicians with interest or expertise in perioperative care and/or mentoring trainees with an interest in perioperative medicine. How can patient outcomes and other metrics like LOS be tracked? What opportunities exist to contribute to research in perioperative medicine?

\section{Consider Creating PPO}

- Ideally an interdisciplinary group effort, get buy-in from key stakeholders

- Standardizes patient care and provides clarification of who to call and when 
Table 1. Establishing a Perioperative Biomarker Screening Program: Commonly Encountered Challenges and Proposed Solutions

Challenge

- Insufficient physicians interested/available to staff a new service, pre-/postoperative clinic, or to accommodate increased workload

- Remuneration insufficient to create a standalone perioperative service

- Issues in arranging timely postoperative clinic follow-up for patients with MINS (or other medical complications).

- Inconsistent practice patterns within disciplines.

- Knowledge deficits of CCS guideline recommendations and/or literature on perioperative risk and role of perioperative biomarkers, evidence, and strategies for intervention for patients with MINS.

- Patient responsibility-MRP

Patient responsibility — evening and weekend coverage (staffing and remuneration)

- Concerns regarding increased LOS with IM involvement

- Timely and reliable biomarker testing (urgent/ emergency patients, transitioning care [and lab orders] from outpatient to inpatient setting).

- Anesthetists may be uncomfortable ordering postoperative troponin testing as they are unable to follow the result once the patient is on the ward.

- Cost and availability of biomarker testing.

- Patients and/or surgeons are uncomfortable with higher risk estimates determined by preoperative BNP/NTproBNP testing.

- Day surgery patients.

\section{Solutions}

- Recruit physicians and/or mentor trainees interested in perioperative care.

- Consider an integrative perioperative service (combination of anesthesia, IM, cardiology, geriatrics, etc.).

- Automatic consults for patients meeting high-risk criteria to ensure that the service is financially viable.

- Integration of a dedicated perioperative NP/PA to prevent overwhelming clinician resources or to allow the physician to schedule concurrent pre-/postoperative clinics.

- NP/PA funding could come from the surgical or shared budgets.

- Educational rounds, journal club, CME events, virtual guest speakers.

- Create PPOs to standardize practice.

- Spending time, and/or arrange electives for trainees, at centers with established perioperative services and biomarker screening programs.

- Provide updates in perioperative care for surgical/medical trainees.

- Attend the annual Perioperative Care Congress to keep up with advances in perioperative medicine, ongoing and future research and trials.

- Determine a shared care model that works for surgical and medical teams.

- Establish consensus on appropriate transfer of care, including revision of existing interservice patient transfers protocols to find a model that satisfies all clinicians and optimizes patient care.

- Agreement from surgical and medicinal services on routine and urgent care in the evening/on weekends.

- Consider a perioperative clinical associate (CA), typically fulfilled by senior residents/fellows; these paid overnight and/or weekend shifts help to offload the admitting on-call team by managing urgent overnight ward issues. Funding could come from surgical, medical, or mixed sources.

- A dedicated perioperative service may assume MRP status for select surgical patients.

- Evidence supports a shorter LOS with shared care models. ${ }^{19,20,23}$

- If concerns regarding LOS are a major obstacle, consider a pilot project to assess the impact on LOS.

- The CCS guidelines recommend against any delay in urgent/emergent surgical cases to perform risk assessment (including BNP/NTproBNP); consider adding BNP/NTproBNP level to preoperative blood work already drawn, or POC testing, for guiding postoperative monitoring needs.

- PPOs help to ensure postoperative troponin testing is ordered appropriately. Once surgery is completed, the anesthetist can highlight if there is an indication for troponin testing and complete, or prompt the surgical team to complete, the PPO.

- Some hospital systems may allow for direct order entry of postoperative troponin.

- There is value in a site-specific cost analysis for BNP/NTproBNP, troponin, and ECG, particularly in contrast to other types of preoperative testing like stress testing or echocardiogram (no longer routinely recommended by CCS guidelines but may still be in practice for some).

- For centers without onsite BNP/NTproBNP testing or without access to timely results, consider POC testing.

- If BNP/NTproBNP testing is unavailable, consider routine postoperative ECG and troponin testing per CCS guidelines. ${ }^{5}$

- The use of BNP/NTproBNP allows for more accurate risk estimates with one in four patients reclassified to a more appropriate risk category of medical (not surgical) complications versus RCRI alone. ${ }^{6}$

- We do not yet have evidence to implement perioperative biomarker use in day surgery patients.

CCS, Canadian Cardiovascular Society; CME, continuous medical eduction; ECG, electrocardiogram; LOS, length of stay; MINS, myocardial injury after noncardiac surgery; MRP, most responsible physician; NP, nurse practitionner; PA, physician assistant; PPO, preprinted order set; POC, point-of-care; RCRI, Revised Cardiac Risk Index 


\section{Summary}

The standard of care for adults undergoing inpatient, noncardiac surgery now includes the use of preoperative BNP/NTproBNP to provide an individualized perioperative risk assessment and routine postoperative troponin monitoring to detect myocardial injury for high-risk patients. Accurate risk prediction is important for informed consent, consideration of alternative surgical strategies, and planning for appropriate postoperative monitoring. Growing evidence demonstrates increased morbidity and mortality of patients who suffer from MINS, an entity that would be largely undetected without biochemical monitoring. ${ }^{2,11}$ Strategies for pharmacotherapy intensification have been shown to improve outcomes for patients with MINS. ${ }^{15,16}$

Clinicians interested in developing a preoperative BNP/ NTproBNP and postoperative troponin screening program will benefit from a site-specific strategy and assessment of clinical, funding, and laboratory resources. This article highlights several successful, yet unique, approaches to establishing sustainable perioperative biomarker monitoring programs. Detailed solutions to commonly encountered challenges have been highlighted along with strong encouragement for the consideration of a multidisciplinary, shared-care model to facilitate the implementation of perioperative biomarker screening, to improve patient outcomes and to increase clinician satisfaction. ${ }^{19-21,24}$

\section{References}

1. Weiser TG, Haynes AB, Molina G, et al. Estimate of the global volume of surgery in 2012: An assessment supporting improved health outcomes. Lancet. 2015 Apr 27;385(Suppl 2):S11. http://dx.doi.org/10.1016/ S0140-6736(15)60806-6

2. Botto F, Alonso-Coello P, Chan MT, Villar JC, Xavier D, Srinathan S, et al. Myocardial injury after noncardiac surgery: A large, international, prospective cohort study establishing diagnostic criteria, characteristics, predictors, and 30-day outcomes. Anesthesiology. 2014;120:564-78. http://dx.doi.org/10.1097/ALN.0000000000000113

3. Fleisher LA, Fleishmann KE, Auerbach AD, et al. 2014 ACC/AHA guideline on perioperative cardiovascular evaluation and management of patients undergoing noncardiac surgery. Circulation. 2014;130:e278-333. http://dx.doi.org/10.1161/CIR.0000000000000104

4. Kristensen SD, Knuuti J, Saraste A, et al. 2014 ESC/ESA guidelines on noncardiac surgery: Cardiovascular assessment and management. Eur Heart J. 2014;35:2383-431. http://dx.doi.org/10.1093/eurheartj/ehu282

5. Duceppe E, Parlow J, MacDonald P, et al. Canadian Cardiovascular Society guidelines on perioperative cardiac risk assessment and management for patients who undergo noncardiac surgery. Can J Cardiol. 2017;33:17-32. http://dx.doi.org/10.1016/j.cjca.2016.09.008

6. Duceppe E, Patel A, Chan MT, Berwanger O, Ackland G, Kavsak PA, et al. Preoperative N-terminal Pro-B-type natriuretic peptide and cardiovascular events after noncardiac surgery: A cohort study. Ann Intern Med. 2020 Jan 21;172(2):96-104. http://dx.doi.org/10.7326/L20-0269

7. Rodseth RN, Biccard BM, Le Manach Y, Sessler DI, Buse GA, Thabane $\mathrm{L}$, et al. The prognostic value of pre-operative and post-operative B-type natriuretic peptides in patients undergoing noncardiac surgery: B-type natriuretic peptide and $\mathrm{N}$-terminal fragment of pro-B-type natriuretic peptide: A systematic review and individual patient data meta-analysis.
J Am Coll Cardiol. 2014 Jan 21;63(2):170-80. http://dx.doi.org/10.1016/j. jacc.2013.08.1630

8. Lee TH, Marcantonio ER, Mangione CM, et al. Derivation and prospective validation of a simple index for prediction of cardiac risk of major noncardiac surgery. Circulation. 1999;100:1043-9. http://dx.doi.org/10.1161/01. CIR.100.10.1043

9. Fleisher LA, Fleischmann KE, Auerbach AD, et al. 2014 ACC/AHA guideline on perioperative cardiovascular evaluation and management of patients undergoing noncardiac surgery: Executive summary: A report of the American College of Cardiology/American Heart Association Task Force on Practice Guidelines. Circulation. 2014;130:2215-45. http://dx.doi. org/10.1161/CIR.0000000000000105

10. Park SJ, Choi JH, Cho SJ, et al. Comparison of transthoracic echocardiography with N-terminal pro-brain natriuretic peptide as a tool for risk stratification of patients undergoing major noncardiac surgery. Korean Circ J. 2011;41:505-11. http://dx.doi.org/10.4070/kcj.2011.41.9.505

11. Devereaux PJ, Biccard BM, Sigamani A, Xavier D, Chan MT, Srinathan SK, et al. Association of postoperative high-sensitivity troponin levels with myocardial injury and 30-day mortality among patients undergoing noncardiac surgery. JAMA. 2017 Apr 25;317(16):1642-51.

12. van Waes JA, Nathoe HM, de Graaff JC, Kemperman H, de Borst GJ, Peelen LM, et al. Myocardial injury after noncardiac surgery and its association with short-term mortality. Circulation. 2013 Jun 11;127(23):2264-71. http://dx.doi.org/10.1161/CIRCULATIONAHA.113.002128

13. Beattie WS, Karkouti K, Tait G, Steel A, Yip P, McCluskey S, et al. Use of clinically based troponin underestimates the cardiac injury in non-cardiac surgery: A single-centre cohort study in 51,701 consecutive patients. Can J Anesth. 2012 Nov 1;59(11):1013-22. http://dx.doi.org/10.1007/ s12630-012-9782-9

14. Devereaux PJ, Szczeklik W. Myocardial injury after non-cardiac surgery: Diagnosis and management. Eur Heart J. 2020 May 1;41(32):3083-91. http://dx.doi.org/10.1093/eurheartj/ehz301

15. Devereaux PJ, Duceppe E, Guyatt G, Tandon V, Rodseth R, Biccard BM, et al. Dabigatran in patients with myocardial injury after non-cardiac surgery (MANAGE): An international, randomised, placebo-controlled trial. Lancet. 2018 Jun 9;391(10137):2325-34.

16. Foucrier A, Rodseth R, Aissaoui M, et al. The long-term impact of early cardiovascular therapy intensification for postoperative troponin elevation after major vascular surgery. Anesth Analg. 2014;119:1053. http://dx.doi. org/10.1213/ANE.0000000000000302

17. Buse GL, Manns B, Lamy A, Guyatt G, Polanczyk CA, Chan MT, et al. Troponin T monitoring to detect myocardial injury after noncardiac surgery: A cost-consequence analysis. Can J Surg. 2018 Jun;61(3):185. http://dx.doi. org/10.1503/cjs.010217

18. Shaw M, Pelecanos AM, Mudge AM. Evaluation of internal medicine physician or multidisciplinary team comanagement of surgical patients and clinical outcomes: A systematic review and meta-analysis. JAMA Netw Open. 2020 May 1;3(5):e204088. http://dx.doi.org/10.1001/ jamanetworkopen.2020.4088

19. Kammerlander C, Roth T, Friedman SM, Suhm N, Luger TJ, KammerlanderKnauer U, et al. Ortho-geriatric service-A literature review comparing different models. Osteoporos Int. 2010 Dec;21(Suppl 4):S637-46. http://dx.doi.org/10.1007/s00198-010-1396-x

20. Grigoryan KV, Javedan H, Rudolph JL. Orthogeriatric care models and outcomes in hip fracture patients: A systematic review and meta-analysis. J Orthop Trauma. 2014 Mar;28(3):e49-55. http://dx.doi.org/10.1097 /BOT.0b013e3182a5a045

21. Huddleston JM, Long KH, Naessens JM, Vanness D, Larson D, Trousdale R, et al. Medical and surgical comanagement after elective hip and knee arthroplasty: A randomized, controlled trial. Ann Intern Med. 2004 Jul 6;141(1):28-38. http://dx.doi. org/10.7326/0003-4819-141-1-200407060-00012

22. Fisher AA, Davis MW, Rubenach SE, Sivakumaran S, Smith PN, Budge MM. Outcomes for older patients with hip fractures: The impact of orthopedic 


\section{Perioperative Medicine Special Issue}

and geriatric medicine cocare. J Orthop Trauma. 2006 Mar;20(3):172-8; discussion 179-80. http://dx.doi.org/10.1097/01.bot.0000202220.88855.16

23. Rohatgi N, Loftus P, Grujic O, Cullen M, Hopkins J, Ahuja N. Surgical comanagement by hospitalists improves patient outcomes. Ann Surg. 2016 Aug 1;264(2):275-82. http://dx.doi.org/10.1097/SLA.0000000000001629

24. McKilligan K. Roche diagnostics, personal communication, November 19, 2020.
25. Roche Diagnostics.. Cardiac markers: Ane tube, standardized POC and laboratory, one complete solution for chest pain management. Laval, QB; 2012.

26. VPSA-funded project aims to improve perioperative cardiac risk evaluation and management for noncardiac surgery [Internet]. VMDAS Association Newsletter. 2018 Oct 16. Available from: https://ourvancouvermsa. ca/2018/10/16/vpsa-funded-project-aims-to-improve-perioperative-cardiacrisk-evaluation-and-management-for-noncardiac-surgery/ 